\title{
Transforming waste polypropylene face masks into S-doped porous carbon as the cathode electrode for supercapacitors
}

\author{
Xiang $\mathrm{Hu}^{1} \cdot$ Zhidan Lin $^{1}$
}

Received: 10 October 2020 / Revised: 27 January 2021 / Accepted: 1 February 2021 / Published online: 18 February 2021

(C) The Author(s), under exclusive licence to Springer-Verlag GmbH, DE part of Springer Nature 2021

\begin{abstract}
The spread of COVID-19 has led to an explosive increase in the number of waste polypropylene face masks worldwide, landfill and incineration of which will cause serious pollution and resource waste. This study aims to develop a new method for the safe and high-added value reuse of materials for polypropylene face masks based on carbonization of porous polymer. The waste masks were first sulfonated in an autoclave, then used as carbon source and turned into a dense hollow fiber porous structure after a one-step heat treatment. This porous structure has a high specific capacitance, namely $328.9 \mathrm{~F} \mathrm{~g}^{-1}$ at a current density of $1 \mathrm{~A}$ $\mathrm{g}^{-1}$. Besides, the assembled solid-state capacitor possesses a good energy density of $10.4 \mathrm{~W} \mathrm{~h} \mathrm{~kg}^{-1}$ at a power density of $600 \mathrm{~W} \mathrm{~kg}^{-1}$, and excellent cycling stability with a capacitance retention rate of $81.1 \%$ after 3000 cycles. These findings indicate that the novel carbonization technology in this study can not only be used to obtain high-performance supercapacitor electrode materials but also provide a new idea for the recycling and utilization of wastes such as medical devices.
\end{abstract}

Keywords Waste mask $\cdot$ Polypropylene $\cdot$ Sulfonation $\cdot$ Carbonization $\cdot$ Supercapacitor $\cdot$ Hollow fiber

\section{Introduction}

At present, COVID-19 epidemic has spread worldwide, the severity of which is unprecedented [1]. During the epidemic, face masks became one of the essential supplies in social interactions [2]. The consumption of face masks has exploded. According to incomplete statistics, about 1.94 trillion face masks and gloves are consumed worldwide each month. With the mass production of face masks, the proposal of waste face masks has become a major problem. Currently, waste face masks cannot be recycled, and most of them are treated by incineration and landfill like ordinary polymer wastes. These methods require high energy cost and cause great damage to the environment [3, 4]. Moreover, face masks directly discarded into dustbins are easily utilized by illegal merchants, resulting in viral transmission. Therefore, it is necessary to increase the added value of waste face masks through safe recycling and utilization. Face mask is mainly made of

Zhidan Lin

linzd@jnu.edu.cn

1 Institute of Advanced Wear \& Corrosion Resistant and Functional Materials, Jinan University, No.601, West Huangpu Avenue, Guangzhou 510632, People's Republic of China polypropylene non-woven fabric, which has a lot of fiber structure and porosity [5]. Therefore, the high-temperature carbonization of face masks can not only inactivate viruses but also be used to prepare porous carbon materials.

Carbonizing waste polymer materials is one of the most promising recycling methods [6]. Carbon is the main component of polymer materials, so carbon sources with added value, such as amorphous carbon (activated carbon, carbon fiber, carbon spheres), graphite, and carbon nanomaterials, can be produced by carbonization [7, 8]. Carbonized products are controllable and energy-saving emission friendly, and do not require complex pretreatment. Many polymer materials have been proven able to produce precursors of carbon materials through carbonization [9]. For example, polyolefins, a plastic waste, can be converted into carbon nanotubes under catalyzed conditions [10,11], and polystyrene waste can be modified by carbonization for supercapacitor electrode materials. LDPE (low-density polyethylene) and HDPE (high-density polyethylene) can be carbonized to form carbon materials for lithium battery electrode materials after sulfonation [12]. Although carbonization based on polypropylene has been studied, the application of preparing electrode materials for supercapacitors is rarely looked into. Carbon materials are usually prepared with $\mathrm{MgO}$ as a template and add catalyst or through chlorination sulfonation modification [13-15]. 
However, these methods bring limited carbon production and have no potential application prospects.

In response to common problems like low carbon yield, low porosity, and low specific capacitance of carbon electrode in carbonization process, this study makes full use of the porous characteristics of polypropylene non-woven mask to prepare high-performance porous carbon materials. Sulfonated waste masks were prepared with solvothermal method in the autoclave, then mixed evenly with activator. Porous carbon materials were obtained after a one-step heat treatment. On the one hand, sulfonation can not only improve the carbon yield but also realize heteroatom doping to improve the physical and chemical properties of porous carbon materials. On the other hand, the pore-forming effect of activator can improve the porosity and enhance the capacitive performance of electrode materials.

\section{Materials and method}

\section{Materials}

Waste polypropylene face masks were collected from mask recycling sites at communities. Concentrated $\mathrm{H}_{2} \mathrm{SO}_{4}(98 \%)$ was obtained from Guangzhou Chemical Reagent Factory (Guangzhou, China). Potassium hydroxide $(\mathrm{KOH})$ was delivered from Damao Chemical Reagent Factory (Tianjin, China). Polyvinyl alcohol (PVA, 1799) was purchased from Shanghai Macklin Biochemical Co., Ltd. (Shanghai, China).

\section{Preparation}

Before preparation, the waste masks were cut into small pieces. Then, $0.5 \mathrm{~g}$ of the waste masks and $10 \mathrm{~mL}$ of sulfuric acid $\left(\mathrm{H}_{2} \mathrm{SO}_{4}\right)$ were mixed and introduced to a Teflon vessel. The mixture-loaded Teflon vessel was put into the autoclave and reacted at $110{ }^{\circ} \mathrm{C}$ for $12 \mathrm{~h}$. After sulfonation, solid black samples were collected and washed with deionized water for several times and dried at $70{ }^{\circ} \mathrm{C}$ for $12 \mathrm{~h}$. Samples collected are named as MS. As-prepared MS was mixed with $\mathrm{KOH}$ in different mass ratios $(1: 1 ; 1: 2 ; 1: 3 ; 1: 4)$, which are named CMS-1, CMS-2, CMS-3, and CMS-4, respectively. After that, the mixture was carbonized and activated at $750{ }^{\circ} \mathrm{C}$ for $2 \mathrm{~h}$ in a tubular furnace under $\mathrm{N}_{2}$ flow with a heating rate of $5{ }^{\circ} \mathrm{C} \mathrm{min}{ }^{-1}$ and then cooled down to room temperature. Black powder was collected and washed with $1 \mathrm{M} \mathrm{HCl}$ solution, washed with deionized water for several times until its $\mathrm{pH}$ value reached about 7 , and then dried at $70^{\circ} \mathrm{C}$ for $12 \mathrm{~h}$.

\section{Characterization}

The morphology and microstructures were analyzed using field-emission scanning microscope (FESEM, Zeiss
ULTRATM 55) and field-emission transmission electron microscopy (FETEM, JEOL JEM-2100F). Element distribution was determined with an energy-dispersive spectrometer (EDS, BRUKER XFlash 6I30). Thermogravimetric analysis (TGA, Mettler Toledo TGA/DSC 3+) was conducted to characterize the decomposition process of samples. Fouriertransform infrared (FTIR, ThermoFisher Nicolet iS50) and Raman spectroscopy (HORIBA LabRAM HR Evolution) were used to further characterize the material's structure. Element valence was conducted with X-ray photoelectron spectroscopy (XPS, Escalab250Xi, Thermo-VG Scientific, MA, USA). The patterns of the carbon samples were determined by X-ray diffraction (XRD, Rigaku Ultima IV instrument) with $\mathrm{Cu} \mathrm{K} \alpha$ radiation at $40 \mathrm{KV}$ and $200 \mathrm{~mA}$.

\section{Electrochemical measurement}

The electrochemical properties of the samples were analyzed via electrochemical impedance spectroscopy (EIS), cyclic voltammetry (CV), and galvanostatic charge/discharge using a $\mathrm{CHI} 760 \mathrm{E}$ workstation $(\mathrm{CH}$ Instruments) in $6 \mathrm{M} \mathrm{KOH}$ electrolyte solution, and with a Pt sheet of $10 * 10 \mathrm{~mm}$ as a counter electrode and a saturated calomel electrode as a reference electrode (SCE). The cycle stability of the samples was characterized by a specific capacitance retention of over 3000 times of repeated charging and discharging. To preparation of the working electrode, we mixed porous carbon electrode material, binder PTFE, and conductive agent acetylene black in a ratio of $8: 1: 1$, coated them on the $1 * 2 \mathrm{~mm}$ foam nickel, then dried and pressed them. The preparation of the solid capacitor was the same as that of the working electrode, but polyvinyl alcohol (PVA)/ $\mathrm{KOH}$ gel was used as the electrolyte.

The calculation formula for the specific capacitance $(C)$ of the working electrode was based on GCD,

$C=\frac{I \Delta t}{m \Delta V}$

where $I(\mathrm{~A})$ is the discharge current, $\Delta t(\mathrm{~s})$ is the discharge time, $m(\mathrm{~g})$ is the mass of active materials in working electrodes, and $\Delta V(\mathrm{~V})$ refers to the discharge voltage excluding the $\mathrm{I}_{\mathrm{R}}$ drop.

The specific capacitance $(C s)$ of the symmetric capacitor was calculated as follows,

$C \mathrm{~s}=\frac{I \Delta t}{m \Delta V}$

where $I(\mathrm{~A})$ is the discharge current, $\Delta t(\mathrm{~s})$ is the discharge time, $m(\mathrm{~g})$ is the total mass of the active materials of cathode and anode, and $\Delta V(\mathrm{~V})$ refers to the potential change in discharge. 


\section{Results and discussion}

\section{Analysis of sulfonated face masks}

The process of preparing CMS (the activated porous carbon) samples is shown in Fig. 1. Generally, waste polypropylene face masks begin to melt at $170{ }^{\circ} \mathrm{C}$. After $400{ }^{\circ} \mathrm{C}$, the polymer chain is gradually broken to produce carbon dioxide, carbon monoxide, low molecular weight alkanes, and other gases. Therefore, it is not easy to obtain high-value carbon materials. To this end, the mask fragments were sulfonated in the autoclave in advance. Hydrothermal reaction contributed to the functionalization of sulfonic acid groups. Due to the strong oxidation of concentrated sulfuric acid, some carbon chains were broken and sulfonic acid groups were introduced. The introduced sulfonic acid groups acted together with $\mathrm{KOH}$, and the carbon skeleton was gradually etched to form a hollow fiber porous structure.

To determine the composition of the waste masks and the presence of sulfonic acid groups, FT-IR was used for spectral analysis and characterization at $400-4000 \mathrm{~cm}^{-1}$ (Fig. 2b). The IR spectrum of the raw materials showed the typical absorption peaks of PP $\left(1370 \mathrm{~cm}^{-1}, 997 \mathrm{~cm}^{-1}\right.$, and $\left.971 \mathrm{~cm}^{-1}\right)$. Because of the spiral arrangement of molecular chain, a series of absorption bands of moderate intensity $\left(1170 \mathrm{~cm}^{-1}\right.$, $997 \mathrm{~cm}^{-1}, 839 \mathrm{~cm}^{-1}$, and $810 \mathrm{~cm}^{-1}$ ) appeared between $1250 \mathrm{~cm}^{-1}$ and $800 \mathrm{~cm}^{-1}$, corresponding to the absorption band of isotactic polypropylene [16]. For MS samples, new characteristic peaks $\left(1180 \mathrm{~cm}^{-1}, 1140 \mathrm{~cm}^{-1}, 587 \mathrm{~cm}^{-1}\right.$, and $518 \mathrm{~cm}^{-1}$ ) appeared in the spectrum due to the incorporation of sulfonic acid groups into the polymer carbon chain of mask materials. Besides, two characteristic peaks $\left(1700 \mathrm{~cm}^{-1}\right.$ and $1610 \mathrm{~cm}^{-1}$ ) appeared, which may result from the formation of carbonyl $(\mathrm{C}=\mathrm{O})$ and carbon-carbon double bonds $(\mathrm{C}=\mathrm{C})$ during sulfonation [13].
To analyze the thermal decomposition of sulfonated face masks, thermogravimetric analysis (TGA) was performed. The thermogravimetric analysis of raw materials for waste mask and the sulfonated mask (MS) was performed at a rate of $10{ }^{\circ} \mathrm{C} \mathrm{min}^{-1}$ under an argon atmosphere to determine carbon yield (Fig. 2a). The TGA curve demonstrated that the raw materials of waste masks began to oxidize and decompose at a temperature higher than $400{ }^{\circ} \mathrm{C}$, and that was almost no solid residue at $480{ }^{\circ} \mathrm{C}$. This indicated that it was difficult to obtain carbon materials for waste masks in an inert gas atmosphere. However, for MS samples obtained by autoclave sulfonation, they started to show mass loss from the beginning. The MS samples gradually changed to carbon-based materials and showed two periods of significant quality degradation. At $100-200{ }^{\circ} \mathrm{C}$, their quality was declined because of the loss of functional (sulfonate) groups. At about $400{ }^{\circ} \mathrm{C}$, due to the oxidative decomposition of some of the raw materials for unsulfonated face mask, their quality degraded rapidly. But overall, carbon yield was still nearly $40 \%$ at a temperature of $900{ }^{\circ} \mathrm{C}$. This may be due to the increased degree of crosslinking of polymer molecules after sulfonation, which improve carbon yield.

\section{Analysis of CMS morphology and structure}

The sulfonation of fibers can form a dense carbon fiber structure [17]. Activation of $\mathrm{KOH}$ can generate new pores and increase total pore volume and specific surface area [18]. Figure $3 \mathrm{a}-\mathrm{b}$ shows the SEM image of CMS-0 (the activated porous carbon with no $\mathrm{KOH}$ ) after carbonization. A dense porous structure is formed on the surface of the hollow fiber. Similar structures were also observed on the morphology of CMS-3 samples (Fig. 3d-e). However, the pore structure on the surface of hollow fiber was denser than that of CMS- 0 and the pore size was smaller than that of CMS- 0 , suggesting that
Fig. 1 Schematic of the formation process of CMS samples. MS is a black solid after sulfonation, while CMS sample is the activated porous carbon

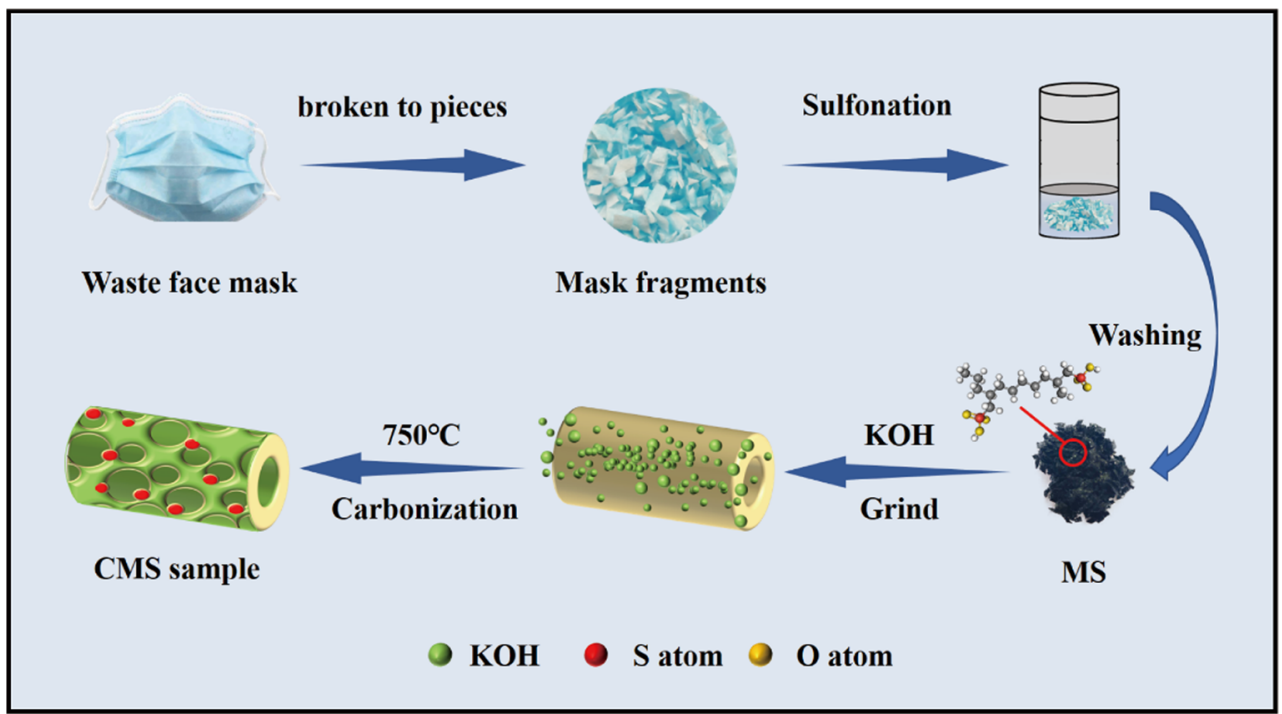



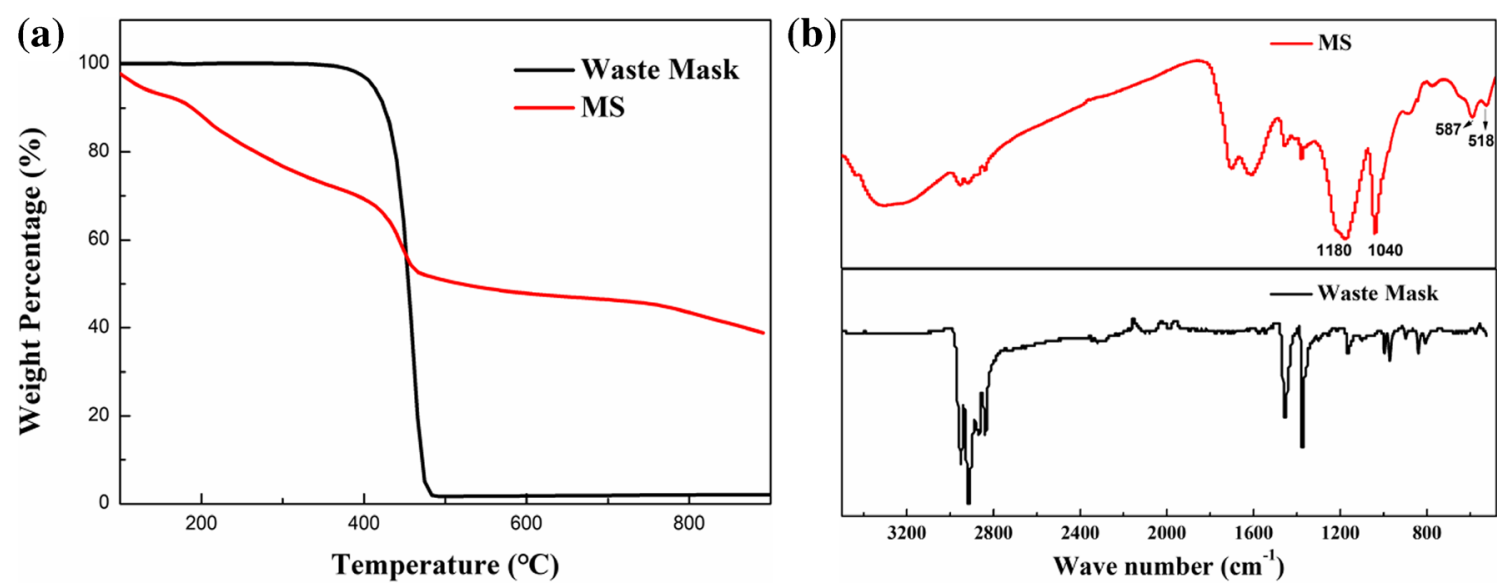

Fig. 2 a TGA analysis of waste mask and sulfonated mask (MS) and b FT-IR spectrum of waste mask and MS

the activation of $\mathrm{KOH}$ can promote the formation of micropore structure. On the one hand, during high-temperature carbonization, the release of gases $\left(\mathrm{CO}, \mathrm{CO}_{2}, \mathrm{H}_{2} \mathrm{O}\right.$, and $\left.\mathrm{SO}_{2}\right)$ may lead to the increase of surface pores and the shrinkage of the diameter of carbon product. On the other hand, due to the etching of the carbon skeleton by $\mathrm{KOH}$, pores on the surface of the carbon skeleton further increased. Simultaneously, their size was contracted, resulting in a dense porous structure. Besides, $\mathrm{C}$, O, and $\mathrm{S}$ in CMS-3 sample were also determined, as shown in Fig. 3e-h. About $88 \%$ wt of C elements were observed by EDS for CMS-3 samples; the rest were $\mathrm{O}$ and $\mathrm{S}$ elements. This may be due to the introduction of $\mathrm{S}$ and $\mathrm{O}$ atoms by sulfonation, realizing heteroatom doping.

The activation of $\mathrm{KOH}$ caused the carbon skeleton to be gradually etched to from a porous carbon structure. Fieldemission transmission electron microscopy (FETEM) image validates the amorphous character of CMS-3 sample with microporous and mesoporous structures (Fig. 4a-b). The selected area electron diffraction (SAED) pattern of CMS-3 sample shows highly diffused diffraction rings without visible spots,
Fig. 3 a, b SEM images with different magnification of CMS$0 ; \mathbf{c}, \mathbf{d}$ SEM images with different magnification of CMS-3; $\mathbf{e}-\mathbf{h}$ EDS mapping of CMS-3
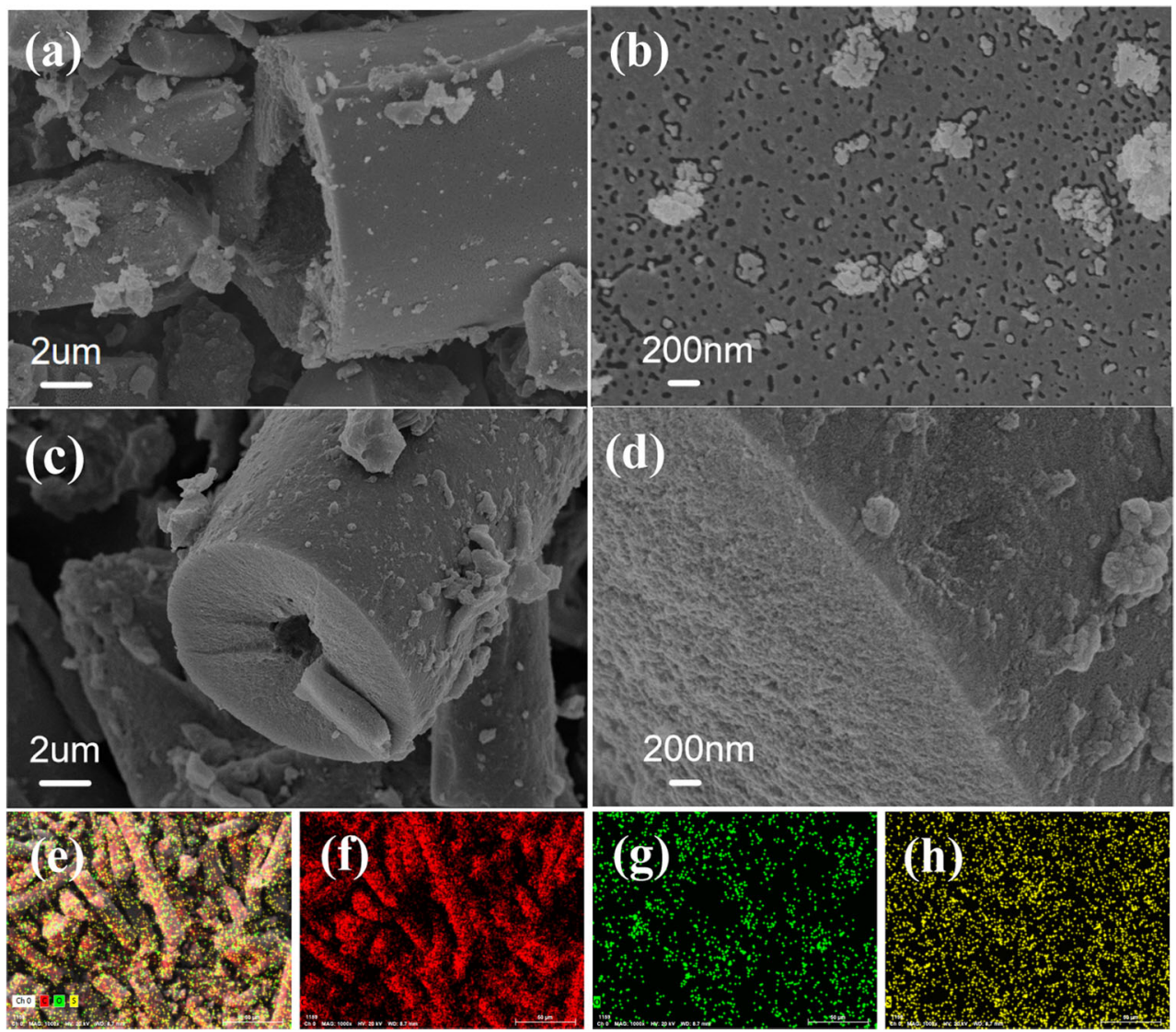
Fig. 4 TEM images of CMS-3
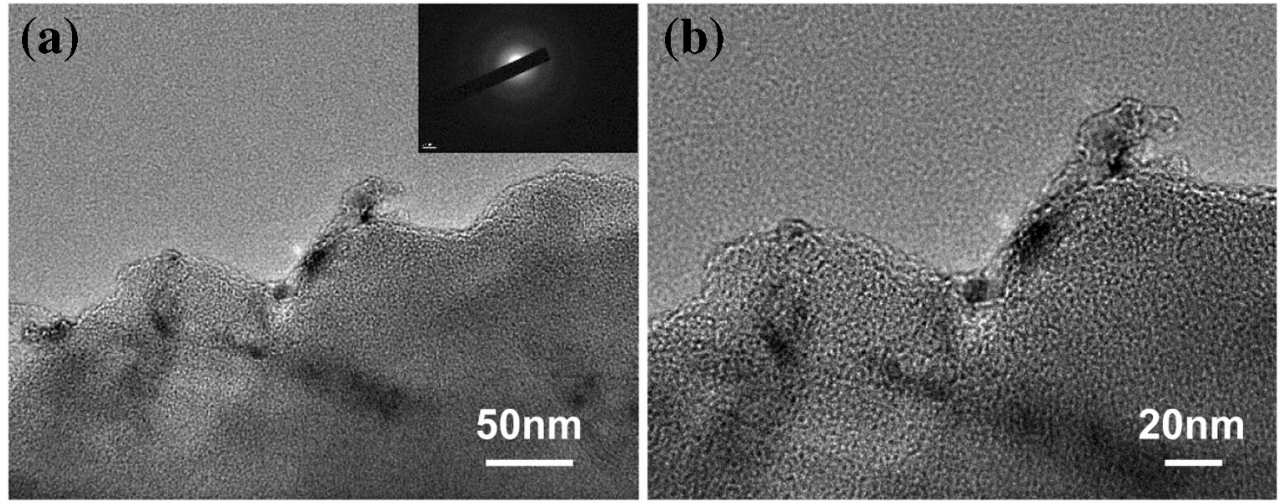

verifying its amorphous character. To further study the crystalline property of CMS samples, powder X-ray diffraction (XRD) test was performed (Fig. 5a). The samples showed a broad peak at $23.6^{\circ}$ and a weak peak at $43.5^{\circ}$, which were characteristic peaks of amorphous graphite carbon, indicating that the carbon material had an amorphous graphite structure. Sulfonic acid groups can induce more sulfur species, and these large-sized sulfur atoms create defects in the carbon skeleton and promote the activation process $[19,20]$. Raman spectroscopy measurement was carried out to further characterize CMS samples (Fig. 5b). The degree of graphitization of the samples can be measured by the $\mathrm{I}_{\mathrm{D}} / \mathrm{I}_{\mathrm{G}}$ ratio. The $\mathrm{D}$ band originates from the lattice defects of $\mathrm{C}$ atom, and the $\mathrm{G}$ band represents the stretching vibration of the sp2 hybrid surface of $\mathrm{C}$ atom. CMS samples can be fitted to 4 peaks at about $1170 \mathrm{~cm}^{-1}, 1330 \mathrm{~cm}^{-1}, 1500 \mathrm{~cm}^{-1}$, and $1600 \mathrm{~cm}^{-1}$, which corresponded to the I, D, D", and G band of carbon, respectively $[21,22]$. The $\mathrm{I}_{\mathrm{D}} / \mathrm{I}_{\mathrm{G}}$ ratios of CMS-0 sample to CMS-4 sample were $1.35,1.38,1.64,1.90$, and 1.92 , respectively. This may be due to $\mathrm{KOH}$ activation destroying the carbon skeleton and increasing the lattice defects of $\mathrm{C}$ atoms.

The specific surface area and porosity of CMS samples were characterized by nitrogen adsorption/desorption experiment. Table 1 summarizes porosity characteristics of CMS samples, and Fig. 6a-b shows CMS samples displayed a combination of type-I and type-IV curves. The $\mathrm{N}_{2}$ isotherms of CMS samples present narrow knees at $\mathrm{P} / \mathrm{P}_{0}<0.1$, indicating the presence of many micropores appearing. A hysteresis loop at $\mathrm{P} / \mathrm{P}_{0}$ in the range of $0.45-1.0$ demonstrates the presence of mesopore structures. With the increase of $\mathrm{KOH}$ content, the proportion of micropores increased, resulting in the specific surface area being gradually increased, because the presence of sulfuric acid groups can induce more defects into carbon framework, promote the activation process, and form a highly interconnected pore structure.

To further study the structure of samples, XPS spectrum was used to analyze CMS-3 to verify the chemical composition and valence state of $\mathrm{C}, \mathrm{O}$, and $\mathrm{S}$ in the CMS-3 sample. The total XPS spectrum of CMS-3 samples (Fig. 7a) showed three peaks at $284.9 \mathrm{eV}$, $532.6 \mathrm{eV}$, and $164.3 \mathrm{eV}$, which corresponded to $\mathrm{C} 1 \mathrm{~s}$, $\mathrm{O} 1 \mathrm{~s}$, and $\mathrm{S} 2 \mathrm{p}$, respectively. The high-resolution $\mathrm{S} 2 \mathrm{p}$ (Fig. 7b) spectrum can be linearly fitted by $164.0 \mathrm{eV}$, $165.2 \mathrm{eV}$, and $168.7 \mathrm{eV}$ [23]. Peak positions at $164.0 \mathrm{eV}$ and $165.2 \mathrm{eV}$ correspond to C-S-C 2p 3/2 and C-S-C 2 p 1/2, respectively. These C-S-C structures exist on the carbon surface and can induce surface polarization to increase the conductivity of carbon bodies. Besides, they can also improve the electrochemical performance of the electrode [24, 25]. The peak at
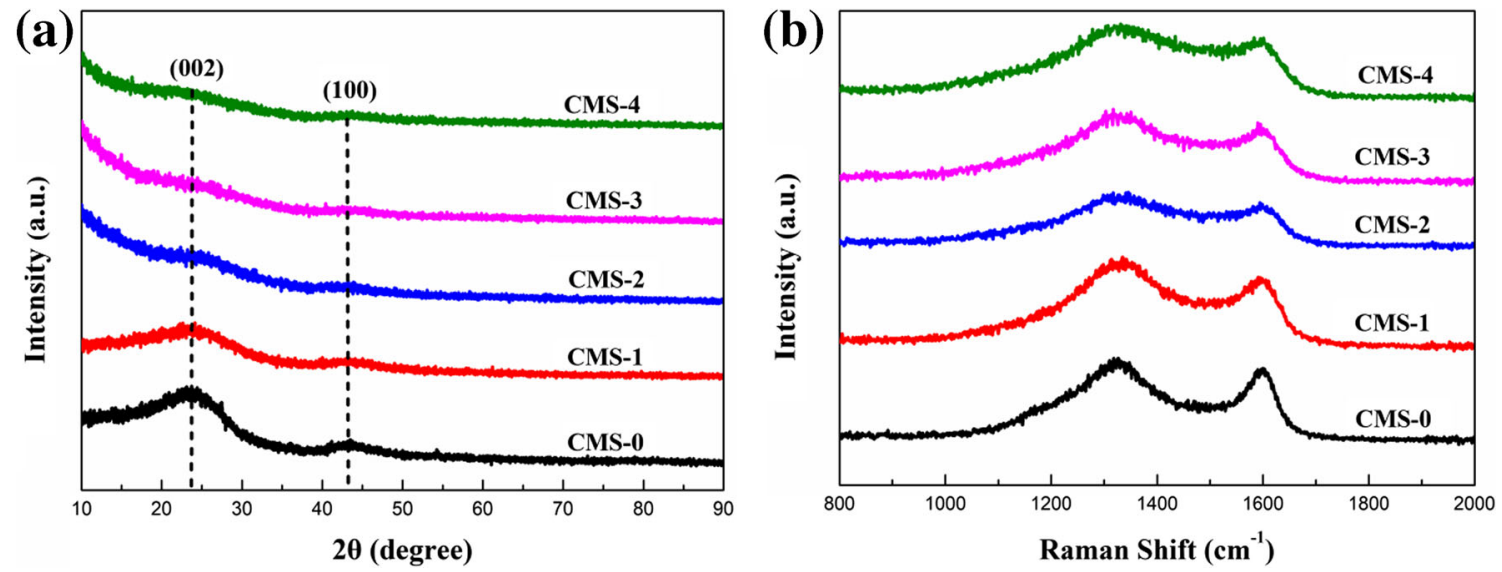

Fig. 5 a XRD patterns of CMS samples and $\mathbf{b}$ Raman spectra of CMS samples 
Table 1 Summary of porosity characteristics of CMS samples

\begin{tabular}{lllll}
\hline Sample & $\mathrm{S}_{\mathrm{BET}}\left[\mathrm{m}^{2} \mathrm{~g}^{-1}\right]$ & $\mathrm{S}_{\text {Languir }}\left[\mathrm{m}^{2} \mathrm{~g}^{-1}\right]$ & $\mathrm{V}_{\mathrm{T}}\left[\mathrm{cm}^{-3} \mathrm{~g}^{-1}\right]$ & $\mathrm{D}_{\text {aver }}[\mathrm{nm}]$ \\
\hline CMS-0 & 505 & 946 & 0.38 & 7.49 \\
CMS-1 & 1146 & 1825 & 0.79 & 8.28 \\
CMS-2 & 1922 & 3053 & 1.10 & 2.42 \\
CMS-3 & 2220 & 3503 & 1.26 & 2.48 \\
CMS-4 & 1768 & 3165 & 1.13 & 2.46 \\
\hline
\end{tabular}

$168.7 \mathrm{eV}$ corresponded to the sulfoxide group connected to the carbon $[26,27]$. And $\mathrm{C} 1 \mathrm{~s}$ (Fig. 7c) can be fitted into three independent peaks, which represented $\mathrm{C}=\mathrm{C}-\mathrm{C}$ $(284.0 \mathrm{eV}), \mathrm{C}-\mathrm{O} / \mathrm{C}-\mathrm{S}(285.4 \mathrm{eV})$, and $\mathrm{C}=\mathrm{O}(288.8 \mathrm{eV})$, respectively, indicating partial graphitization [24]. For O 1s (Fig. 7d), there were two independent peaks at $531.0 \mathrm{eV}$ and $532.0 \mathrm{eV}$, indicating oxygen atoms in CMS-3 samples [20].

\section{Electrochemical measurement}

At room temperature, cyclic voltammetry (CV) was used to characterize the electrochemical performance of CMS samples in a $6 \mathrm{M} \mathrm{KOH}$ electrolyte (Fig. 8a). All samples presented an approximately standard rectangular pattern between $-0.8 \mathrm{~V}$ and $0 \mathrm{~V}$, and showed a typical double-layer charge and discharge process. Generally, the larger the rectangular area enclosed by the $\mathrm{CV}$ curve, the higher specific capacitance of the electrode material. The figure shows CMS-3 samples had a higher response current at the same scanning speed, and that their shape was closer to a standard rectangle. The rectangular area of CMS-3 samples was larger, so we can infer that CMS-3 had a higher specific capacitance, which also indicated that CMS-3 samples had an ideal double-layer capacitance behavior. To further explore the electrochemical performance of CMS-3 samples, cyclic voltammetry test (Fig. 8b) was performed on the
CMS-3 sample at a scan rate of $10-200 \mathrm{mV} \mathrm{s}^{-1}$. The results showed that CMS-3 samples had similar rectangular shapes at different scan rates, which means CMS3 samples had excellent rate capability, as also demonstrated in Fig. 8c.

Figure $8 \mathrm{c}-\mathrm{d}$ studies the constant current charging and discharging process of CMS samples. The curves were similar to the isosceles triangle, demonstrating the electric double-layer capacitance characteristics of CMS samples. Figure $8 \mathrm{~d}$ is the constant current charge and discharge curve of CMS-3 samples at different current densities. CMS-3 samples possess high specific capacitance of $365.4 \mathrm{~F} \mathrm{~g}^{-1}, 328.9 \mathrm{~F} \mathrm{~g}^{-1}, 298.3 \mathrm{~F} \mathrm{~g}^{-1}$, $288.0 \mathrm{~F} \mathrm{~g}^{-1}, 276.0 \mathrm{~F} \mathrm{~g}^{-1}$, and $259.2 \mathrm{~F} \mathrm{~g}^{-1}$ at $0.5 \mathrm{~A}$ $\mathrm{g}^{-1}, 1 \mathrm{~A} \mathrm{~g}^{-1}, 2 \mathrm{~A} \mathrm{~g}^{-1}, 3 \mathrm{~A} \mathrm{~g}^{-1}, 5 \mathrm{~A} \mathrm{~g}^{-1}$, and $10 \mathrm{~A} \mathrm{~g}^{-1}$, respectively. Their capacitance retention rate was $70.9 \%$. The reason for the rise in specific capacitance may be mainly due to activation of $\mathrm{KOH}$, which transforms the carbon structure into a porous structure and accelerates the rapid diffusion of ions [27]. Besides, when sulfur atoms enter the carbon skeleton, the doping process provides more electrochemically active zones and enhances ion transport characteristics [26, 28]. The choice of activator is very important in controlling specific surface area and pore size distribution. The mixing of the different mass ratios of $\mathrm{KOH}$ has a great influence on porous carbon material. Figure $8 \mathrm{c}$ shows the constant current charge and discharge curve with different mass ratios of $\mathrm{KOH}$. After adding $\mathrm{KOH}$, the surface of the carbon skeleton formed a porous structure, which promoted the transfer and diffusion of electrolyte ions. As the concentration of $\mathrm{KOH}$ increased, the activation reaction became more and more complete. Because the carbon skeleton was gradually etched, the proportion of micro-mesopores increased, and the specific surface area and pore diameter gradually reached the maximum value. When the ratio exceeded 4:1 (KOH:MS), the specific capacitance and other electrochemical properties
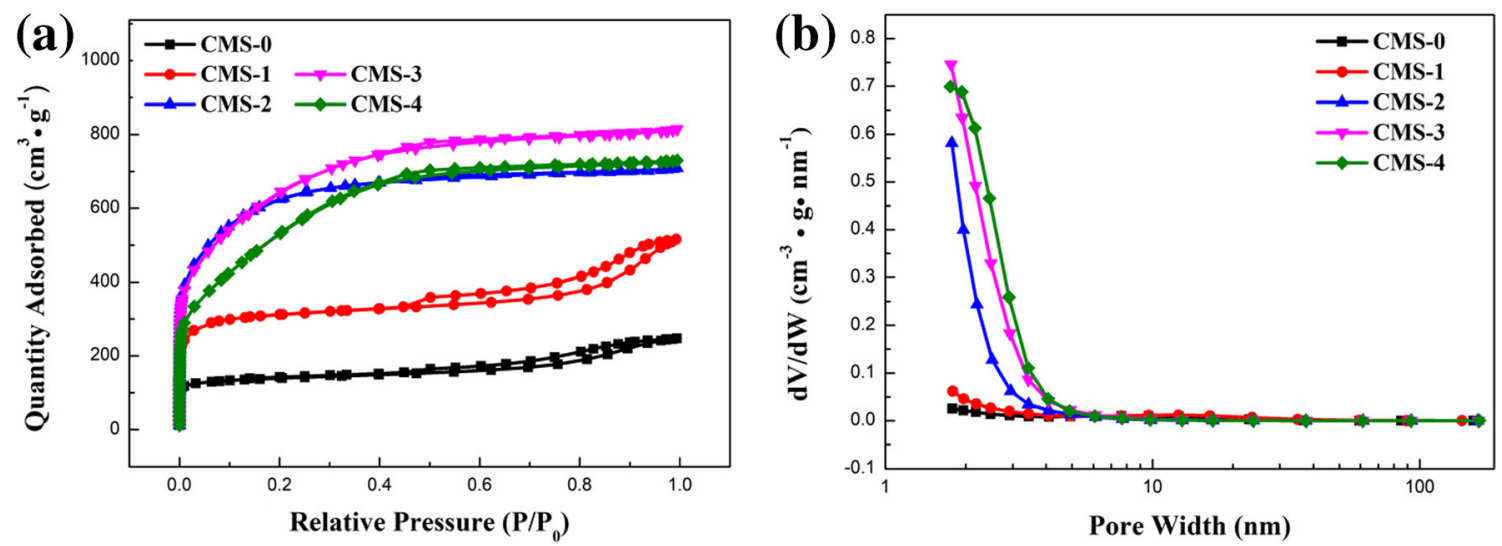

Fig. 6 a $\mathrm{N}_{2}$ adsorption/desorption isotherms and $\mathbf{b}$ pore size distributions of CMS samples 

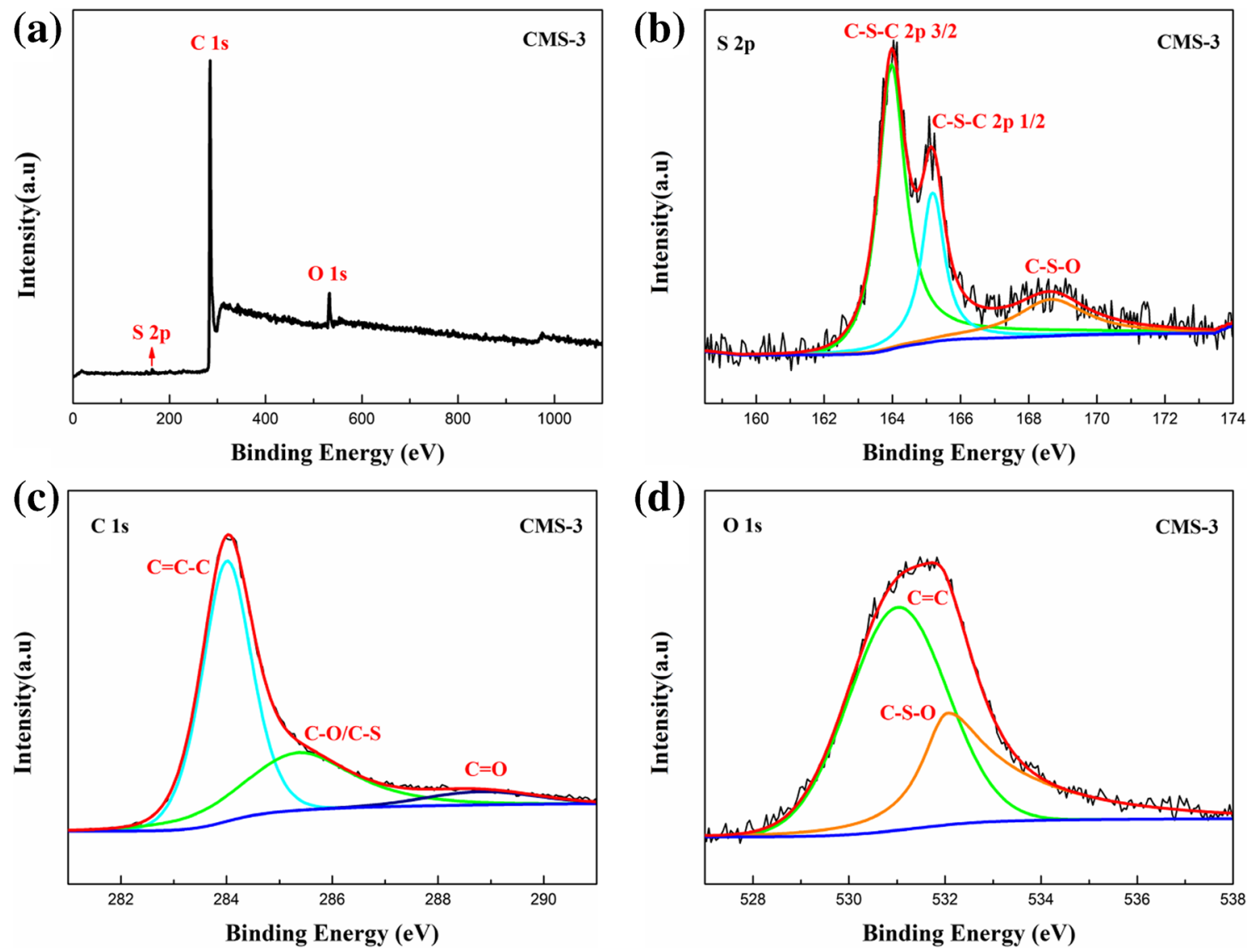

Fig. 7 a The total XPS spectra of CMS-3; b S 2p and $\mathbf{c}$ C 1 s and $\mathbf{d} \mathrm{O} 1 \mathrm{~s}$

began to decrease. This may be caused by excessive ablation of carbon skeleton due to excessive $\mathrm{KOH}$, which collapsed the carbon skeleton around some micropores, causing some micropores to connect into mesopores or even macropores. The specific surface area and pore size of carbon materials decreased.

To further study the electrochemical performance of CMS-3 sample, EIS analysis was conducted. From Fig. $8 \mathrm{e}$, it can be seen that the typical Nyquist plot is composed of high-frequency regions and low-frequency regions. Nyquist plot at high-frequency regions corresponds to the ionic charge transfer resistance formed between the electrolyte and the electrode. And Nyquist plot at low-frequency regions is approximately vertical lines, indicating the pure capacitive behavior of the carbon material and confirming the existence of micronano structures with low resistance and rapid ion transport [34]. The long-term stability was tested by 10,000 cycles at a current density of $15 \mathrm{~A} \mathrm{~g}^{-1}$ (Fig. 8f). After 10,000 cycles, the capacitance retention rate and coulombic efficiency were $90.4 \%$ and $97.0 \%$, respectively. Table 2 summarizes the research reports of some waste materials as supercapacitor materials, in which CMS-3 samples have excellent electrochemical performance.

To further study the practical application of the CMS3 electrode in energy storage, a solid symmetrical capacitor (S-CMS-3) was fabricated. The solid-state symmetrical capacitor is composed of two CMS-3 electrodes with $\mathrm{PVA} / \mathrm{KOH}$ gel as the electrolyte. The $\mathrm{CV}$ curve of the solid-state capacitor device (Fig. 9a) still maintained a rectangular shape at different scan speeds of 10 $200 \mathrm{mV} \mathrm{s}^{-1}$, which is a characteristic of a typical electric double-layer capacitor. Figure 9e shows the Nyquist plot of the device with a shape close vertical, suggesting low impedance. The slope of straight line is related to the diffusion limitation of electrolyte ions (Warburg impedance, $\mathrm{W}$ ), and the semicircular radius can be indexed to the charge transfer resistance (Rct). Nyquist plot at lowfrequency regions is approximately vertical lines and Rct value, indicating best ions diffusion and fast charge transfer rate performance. After the solid-state capacitor device is assembled, the charge transfer resistance between the electrode and the electrolyte increased, which reduced additional $\mathrm{I}_{R}$ and specific capacitance [35]. Figure $9 \mathrm{~b}$ are curves of constant current charging and 

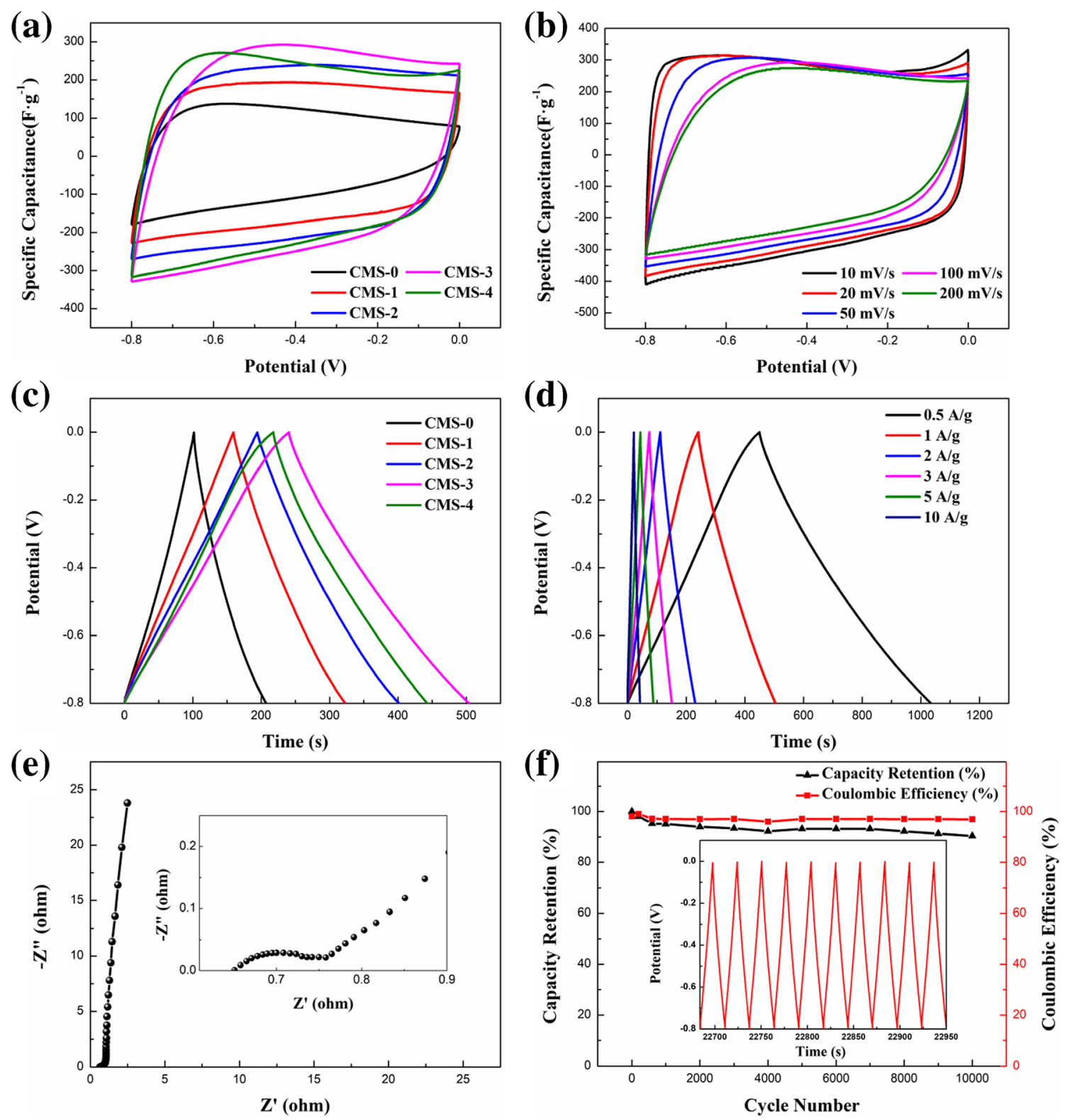

Fig. 8 Electrochemical performance of CMS. a CV curves of CMS with different contents of $\mathrm{KOH}$ at $100 \mathrm{mV} \mathrm{s}^{-1}$; b CV curves of CMS-3 at different scan rates; $\mathbf{c}$ charge-discharge curves of CMS with different

contents of $\mathrm{KOH}$ at $1 \mathrm{~A} \mathrm{~g}^{-1}$; d charge-discharge curves of CMS-3 at different current densities; e Nyquist plot of CMS-3; and $\mathbf{f}$ capacity retention and coulombic efficiency of CMS-3 electrode after 10,000 cycles

discharging at different current densities. The assembled specific capacitance was $52.1 \mathrm{~F} \mathrm{~g}^{-1}$ at $1 \mathrm{~A} \mathrm{~g}^{-1}$ (Fig. 9c). Energy density and long-term stability are important parameters for the practical application of solid-state capacitors. Energy density and power density were calculated according to the constant current charge and discharge curve (Fig. 9d). When the power density is $300 \mathrm{~W} \mathrm{~kg}^{-1}$, the energy density is $11.2 \mathrm{~W} \mathrm{~h} \mathrm{~kg}^{-1}$. When the power density is $6000 \mathrm{~W} \mathrm{~kg}^{-1}$, the energy density is $7.68 \mathrm{~W} \mathrm{~h} \mathrm{~kg} \mathrm{~W}^{-1}$. The long-term stability was tested by 3000 cycles at a current density of $5 \mathrm{~A} \mathrm{~g} \mathrm{~g}^{-1}$
(Fig. 9f). After 3000 cycles, the capacitance retention rate coulombic efficiencies were $81.1 \%$ and $93.0 \%$.

\section{Conclusion}

In summary, new carbon electrode material with a dense hollow fiber porous structure was successfully prepared by combining sulfonation and carbonization method. The resulting carbon electrode materials can reach a high specific capacitance with $328.9 \mathrm{~F} \mathrm{~g}^{-1}$ at $1 \mathrm{~A} \mathrm{~g}^{-1}$ and have been successfully 
Table 2 Comparison of specific capacitances for CMS sample with carbon materials in relevant reports

\begin{tabular}{|c|c|c|c|c|c|c|}
\hline Sample name & Raw material & Specific capacity & Electrolyte & $\begin{array}{l}\text { Power density } \\
\text { (device) }\end{array}$ & $\begin{array}{l}\text { Energy density } \\
\text { (device) }\end{array}$ & Reference \\
\hline HPC-4 & Waste PET bottles & $413 \pm 19 \mathrm{~F} \cdot \mathrm{g}^{-1} / 1 \mathrm{~A} \cdot \mathrm{g}^{-1}(-1-0 \mathrm{~V})$ & $6 \mathrm{M} \mathrm{KOH}$ & $450 \mathrm{~W} \cdot \mathrm{kg}^{-1}$ & $25 \mathrm{~W} \cdot \mathrm{h} \cdot \mathrm{kg}^{-1}$ & [29] \\
\hline PSC-3-700 & Expanded polystyrene foam & $327 \mathrm{~F} \cdot \mathrm{g}^{-1} / 1 \mathrm{~A} \cdot \mathrm{g}^{-1}(-0.2-0.8 \mathrm{~V})$ & $1 \mathrm{M} \mathrm{H}_{2} \mathrm{SO}_{4}$ & $250 \mathrm{~W} \cdot \mathrm{kg}^{-1}$ & $10.2 \mathrm{~W} \cdot \mathrm{h} \cdot \mathrm{kg}^{-1}$ & [30] \\
\hline LPCD-30 & Waste lignin & $325 \mathrm{~F} \cdot \mathrm{g}^{-1} / 1 \mathrm{~A} \cdot \mathrm{g}^{-1}(-1-0 \mathrm{~V})$ & $6 \mathrm{M} \mathrm{KOH}$ & $100 \mathrm{~W} \cdot \mathrm{kg}^{-1}$ & $8.4 \mathrm{~W} \cdot \mathrm{h} \cdot \mathrm{kg}^{-1}$ & [31] \\
\hline LS-800 & Loofah sponge & $304 \mathrm{~F} \cdot \mathrm{g}^{-1} / 1 \mathrm{~A} \cdot \mathrm{g}^{-1}(-1-0 \mathrm{~V})$ & $6 \mathrm{M} \mathrm{KOH}$ & $500 \mathrm{~W} \cdot \mathrm{kg}^{-1}$ & $10 \mathrm{~W} \cdot \mathrm{h} \cdot \mathrm{kg}^{-1}$ & [18] \\
\hline NPCs-2-700 & Melamine-urea-formaldehyde resins & $341 \mathrm{~F} \cdot \mathrm{g}^{-1} / 1 \mathrm{~A} \cdot \mathrm{g}^{-1}(-0.2-(-0.8) \mathrm{V})$ & $6 \mathrm{M} \mathrm{KOH}$ & $350.15 \mathrm{~W} \cdot \mathrm{kg}^{-1}$ & $9.6 \mathrm{~W} \cdot \mathrm{h} \cdot \mathrm{kg}^{-1}$ & [32] \\
\hline KOH-1 & Cellulose & $162 \mathrm{~F} \cdot \mathrm{g}^{-1} / 0.5 \mathrm{~A} \cdot \mathrm{g}^{-1}(-1-0 \mathrm{~V})$ & $6 \mathrm{M} \mathrm{KOH}$ & $5532 \mathrm{~W} \cdot \mathrm{kg}^{-1}$ & $4.1 \mathrm{~W} \cdot \mathrm{h} \cdot \mathrm{kg}^{-1}$ & [33] \\
\hline CMS-3 & Waste face mask & $328.9 \mathrm{~F} \cdot \mathrm{g}^{-1} / 1 \mathrm{~A} \cdot \mathrm{g}^{-1}(-0.8-0 \mathrm{~V})$ & $6 \mathrm{M} \mathrm{KOH}$ & $300 \mathrm{~W} \cdot \mathrm{kg}^{-1}$ & $11.2 \mathrm{~W} \cdot \mathrm{h} \cdot \mathrm{kg}^{-1}$ & This wor \\
\hline
\end{tabular}
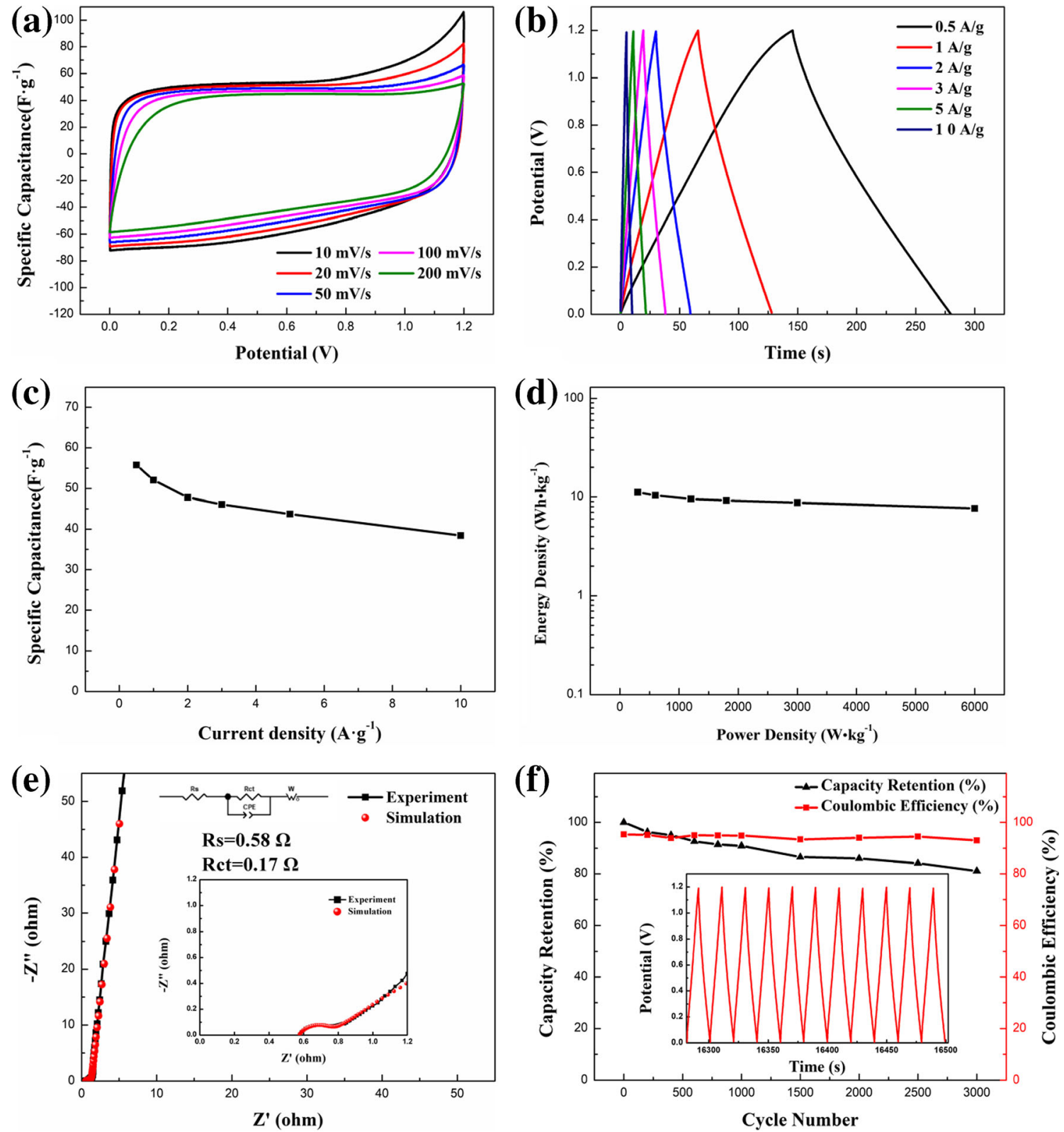

Fig. 9 Electrochemical performance of S-CMS-3. a CV curves of SCMS-3 at different scan rates; $\mathbf{b}$ charge-discharge curves of S-CMS-3 at different current densities; c specific capacitance of S-CMS-3 at

different current densities; $\mathbf{d}$ energy and power density of S-CMS-3; e Nyquist plot of S-CMS-3; and $\mathbf{f}$ capacity retention and coulombic efficiency of S-CMS-3 electrode after 3000 cycles 
applied to supercapacitor. The solid-state capacitor assembled by CMS-3 and PVA/KOH possesses a high capacitance of $52.1 \mathrm{~F} \mathrm{~g} \mathrm{~g}^{-1}$ at $1 \mathrm{~A} \mathrm{~g} \mathrm{~g}^{-1}$, and a good energy density of $10.4 \mathrm{~W} \mathrm{~h} \mathrm{~kg}^{-1}$ at a power density of $600 \mathrm{~W} \mathrm{~kg}^{-1}$. Due to the comprehensive effect of sulfonic acid groups and $\mathrm{KOH}$, the carbon surface forms a dense porous structure, promoting the rapid diffusion of ions. The unique sulfonated sulfur doping carbonization technology has high carbon yield and good capacitive performance of the prepared carbon electrode. This method can provide a new idea for the safe treatment and high added value resource utilization of other polymer-based medical wastes.

Funding This work was supported by the Guang Dong Tobacco Monopoly Administration Science and Technology Programs (no.2019011).

\section{Declarations}

Competing interests The authors declare no competing interests.

\section{References}

1. Garcia-Basteiro AL, Chaccour C, Guinovart C, Llupia A, Brew J, Trilla A, Plasencia A (2020) Monitoring the COVID-19 epidemic in the context of widespread local transmission. Lancet Respir Med 8:440-442

2. Feng S, Shen C, Xia N, Song W, Fan MZ, Cowling BJ (2020) Rational use of face masks in the COVID-19 pandemic. Lancet Respir Med 8:434-436

3. Fonseca WS, Meng XH, Deng D (2015) Trash to treasure: transforming waste polystyrene cups into negative electrode materials for sodium ion batteries. ACS Sustain Chem Eng 3:21532159

4. Pol VG (2010) Upcycling: converting waste plastics into paramagnetic, conducting, solid, pure carbon microspheres. Environ Sci Technol 44:4753-4759

5. Chellamani K, Veerasubramanian D, Balaji RV (2013) Surgical face masks: manufacturing methods and classification. J Acad Indust Res 2:320-324

6. Min JK, Zhang S, Li JX, Klingeler R, Wen X, Chen XC, Zhao X, Tang T, Mijowska E (2019) From polystyrene waste to porous carbon flake and potential application in supercapacitor. Waste Manag 85:333-340

7. Bazargan A, McKay G (2012) A review - synthesis of carbon nanotubes from plastic wastes. Chem Eng J 195:377-391

8. Chen SL, Liu Z, Jiang SH, Hou HQ (2020) Carbonization: a feasible route for reutilization of plastic wastes. Sci Total Environ 710: 19

9. Zhuo CW, Levendis YA (2014) Upcycling waste plastics into carbon nanomaterials: a review. J Appl Polym Sci 131:14

10. Kong QH, Zhang JH (2007) Synthesis of straight and helical carbon nanotubes from catalytic pyrolysis of polyethylene. Polym Degrad Stab 92:2005-2010

11. Tang T, Chen XC, Meng XY, Chen H, Ding YP (2005) Synthesis of multiwalled carbon nanotubes by catalytic combustion of polypropylene. Angew Chem Int Ed 44:1517-1520
12. Villagomez-Salas S, Manikandan P, Guzman SFA, Pol VG (2018) Amorphous carbon chips li-ion battery anodes produced through polyethylene waste upcycling. Acs Omega 3:17520-17527

13. Karacan I, Benli H (2012) Use of sulfonation procedure for the development of thermally stabilized isotactic polypropylene fibers prior to carbonization. J Appl Polym Sci 123:234-245

14. Ma JL, Liu J, Song JF, Tang T (2018) Pressurized carbonization of mixed plastics into porous carbon sheets on magnesium oxide. RSC Adv 8:2469-2476

15. Song CY, Hao L, Zhang BY, Dong ZY, Tang QQ, Min JK, Zhao Q, Niu R, Gong J, Tang T (2020) High-performance solar vapor generation of $\mathrm{Ni}$ /carbon nanomaterials by controlled carbonization of waste polypropylene. Sci China Mater 63:779-793

16. Tao R, Pang QY, Xu T, Xu LY, Yang XH, Zhu XS (2020) Microstructure and sulfonation of dual monomer-grafted polypropylene nonwoven fabrics. J Appl Polym Sci 137:11

17. Xie B, Hong L, Chen P, Zhu B (2016) Effect of sulfonation with concentrated sulfuric acid on the composition and carbonizability of LLDPE fibers. Polym Bull 73:891-908

18. LuanYT WL, Guo SE, Jiang BJ, Zhao DD, Yan HJ, Tian CG, Fu HG (2015) A hierarchical porous carbon material from a loofah sponge network for high performance supercapacitors. RSC Adv 5:42430-42437

19. Zhou J, Shen HL, Li ZH, Zhang S, Zhao YT, Bi X, Wang YS, Cui HY, Zhuo SP (2016) Porous carbon materials with dual N, Sdoping and uniform ultra-microporosity for high performance supercapacitors. Electrochim Acta 209:557-564

20. Deng QD, Abbas SC, Li ZL, Lv JQ, Ma XJ, Cao SL, Ni YH, Zhao WG (2020) Chemically modified self-doped biocarbon via novel sulfonation assisted sacrificial template method for high performance flexible all solid-state supercapacitor. J Colloid Interface Sci 574:33-42

21. Liu Y, Sun ZH, Sun X, Lin Y, Tan K, Sun JF, Liang LW, Hou LR, Yuan CZ (2020) Construction of hierarchical nanotubes assembled from ultrathin $\mathrm{V}_{3} \mathrm{~S}_{4} @ \mathrm{C}$ nanosheets towards alkali-ion batteries with ion-dependent electrochemical mechanisms. Angew Chem Int Ed 59:2473-2482

22. Vallerot JM, Bourrat X, Mouchon A, Chollon G (2006) Quantitative structural and textural assessment of laminar pyrocarbons through Raman spectroscopy, electron diffraction and few other techniques. Carbon 44:1833-1844

23. Wang B, Ji LL, Yu YL, Wang NX, Wang J, Zhao JB (2019) A simple and universal method for preparing $\mathrm{N}, \mathrm{S}$ co-doped biomass derived carbon with superior performance in supercapacitors. Electrochim Acta 309:34-43

24. Balaji SS, Raj JA, Karnan M, Sathish M (2019) Supercritical fluid assisted synthesis of S-doped graphene and its symmetric supercapacitor performance evaluation using different electrolytes. Synth Met 255:9

25. Deng WF, Zhang YJ, Yang L, Tan YM, Ma M, Xie QJ (2015) Sulfur-doped porous carbon nanosheets as an advanced electrode material for supercapacitors. RSC Adv 5:13046-13051

26. Bandosz TJ, Ren TZ (2017) Porous carbon modified with sulfur in energy related applications. Carbon 118:561-577

27. Parveen N, Ansari MO, Ansari SA, Cho MH (2016) Simultaneous sulfur doping and exfoliation of graphene from graphite using an electrochemical method for supercapacitor electrode materials. J Mater Chem A 4:233-240

28. Wang C, Wang HW, Dang BK, Wang Z, Shen XP, Li CC, Sun QF (2020) Ultrahigh yield of nitrogen doped porous carbon from biomass waste for supercapacitor. Renew Energy 156:370-376

29. Liu XG, Wen YL, Chen XC, Tang T, Mijowska E (2020) Coetching effect to convert waste polyethylene terephthalate into hierarchical porous carbon toward excellent capacitive energy storage. Sci Total Environ 723:11 
30. Deka N, Barman J, Kasthuri S, Nutalapati V, Dutta GK (2020) Transforming waste polystyrene foam into $\mathrm{N}$-doped porous carbon for capacitive energy storage and deionization applications. Appl Surf Sci 511:10

31. Chen WM, Luo M, Yang K, Zhou XY (2020) Microwave-assisted $\mathrm{KOH}$ activation from lignin into hierarchically porous carbon with super high specific surface area by utilizing the dual roles of inorganic salts: microwave absorber and porogen. Microporous Mesoporous Mater 300:110178

32. Yu J, Fu N, Zhao J, Liu R, Li F, Du YC, Yang ZL (2019) High specific capacitance electrode material for supercapacitors based on resin-derived nitrogen-doped porous carbons. ACS Omega 4: 15904-15911

33. Abouelamaiem DI, He GJ, Parkin I, Neville TP, Jorge AB, Ji S, Wang RF, Titirici MM, Shearing PR, Brett DJL (2018) Synergistic relationship between the three-dimensional nanostructure and electrochemical performance in biocarbon supercapacitor electrode materials. Sustain Energy Fuels 2:772-785

34. Fang ZM, Cao L, Lai FL, Kong DB, Du XS, Lin HJ, Lin ZD, Zhang P, Li W (2019) Carbon nano bowl array derived from a corncob sponge/carbon nanotubes/polymer composite and its electrochemical properties. Compos Sci Technol 183:8

35. Lv JQ, Zeng P, Abbas SC, Guan XF, Luo PH, Chen DG, Wang YB (2019) Electrochemically scalable production of bilayer fluorographene nanosheets for solid-state supercapacitors. J Mater Chem A 7:16876-16882

Publisher's note Springer Nature remains neutral with regard to jurisdictional claims in published maps and institutional affiliations. 